Article

\title{
Location-Aware Node Management Solution for Multi-Radio Dual Connectivity Scenarios
}

\author{
Jesús Burgueño*D, Isabel de-la-Bandera (D) and Raquel Barco (D) \\ Instituto Universitario de Investigación en Telecomunicación (TELMA), Universidad de Málaga, CEI Andalucía \\ TECH, 29010 Málaga, Spain; ibanderac@ic.uma.es (I.d.-1.-B.); rbm@ic.uma.es (R.B.) \\ * Correspondence: jesusbr@ic.uma.es
}

Citation: Burgueño, J.;

de-la-Bandera, I.; Barco, R.

Location-Aware Node Management

Solution for Multi-Radio Dual

Connectivity Scenarios. Sensors 2021,

21, 7450. https://doi.org/10.3390/

s21227450

Academic Editor: Aboelmagd

Noureldin

Received: 11 October 2021

Accepted: 8 november 2021

Published: 9 November 2021

Publisher's Note: MDPI stays neutral with regard to jurisdictional claims in published maps and institutional affiliations.

\begin{abstract}
The location of user equipments (UEs) allows application developers to customize the services for users to perceive an enhanced experience. In addition, this UE location enables network operators to develop location-aware solutions to optimize network resource management. Moreover, the combination of location-aware approaches and new network features introduced by 5G enables to further improve the network performance. In this sense, dual connectivity (DC) allows users to simultaneously communicate with two nodes. The basic strategy proposed by 3GPP to select these nodes is based only on the power received by the users. However, the network performance could be enhanced if an alternative methodology is proposed to make this decision. This paper proposes, instead of power-based selection, to choose the nodes that provide the highest quality of experience (QoE) to the user. With this purpose, the proposed system uses the UE location as well as multiple network metrics as inputs. A dense urban scenario is assumed to test the solution in a system-level simulation tool. The results show that the optimal selection varies depending on the UE location, as well as the increase in the QoE perceived by users of different services.
\end{abstract}

Keywords: multi-radio dual connectivity; location-awareness; cellular network node management

\section{Introduction}

The position tracking of mobile network users has enabled to develop a wide variety of new applications nowadays, from assisted driving services [1] to the customization of points of interest recommendation [2]. In these cases, users share their location to receive more advanced services. However, the network operator can also use the location of the user equipments (UEs) to enhance their condition within the mobile network, improving the quality of experience $(\mathrm{QoE})$ perceived in the applications they use. For example, the user location can be used to detect and manage network failures by generating new indicators about areas of interest [3] or to decrease the interference level by adjusting the transmission power $[4,5]$. These features can increase the overall performance obtained in the mobile network, which can lead users to perceive a better QoE because the increased network availability or the higher average Signal-to-Interference-plus-Noise Ratio (SINR).

Moreover, the relevance of the location-awareness functionalities acquire a greater magnitude when combined with new fifth-generation (5G) technologies deployed on nowadays non-standalone networks [6]. For example, the authors of [7] use the location of users to noticeably decrease the interference level when beamforming is used. In a similar context, multi-radio dual connectivity (MR-DC) is one of the $5 \mathrm{G}$ features that allows more flexibility in network resource management on non-standalone networks. It allows UEs to simultaneously exchange data with one eNB (evolved Node B) and one gNB (5G New Radio node B), i.e., two nodes that belong to $4 \mathrm{G}$ and 5G, respectively [8]. Two different roles will be assigned to these nodes. One node is selected as master, which exchanges signalling between the UE and the core network $(\mathrm{CN})$, as well as defining the radio resource control (RRC) state. In addition, the master node also sends data to the UE. On the other hand, the other node will be assigned as secondary role, only delivering data to the UE. 
Among other benefits, MR-DC allows UEs to utilize more capacity to reach higher data rates or, alternatively, to send the same information through both links to increase the communication reliability. For instance, the simultaneous transmission of the same data packet through multiple links is addressed in [9]. In this way, it allows to improve the communication reliability as well as latency. Moreover, a mechanism that decides the transmissions to be duplicated is introduced in [10]. It further improves the spectral efficiency. On the other hand, the uneven allocation of resources of the serving nodes allows optimizing the network performance for different purposes. In this way, the authors of [11] propose that each node provides different flow of data in order to minimize the user energy consumption. Similarly, the scheme proposed in [12] pursues a compromise between the minimum energy consumption of the serving nodes and the highest possible Quality of Service (QoS) provided to the UE. The authors of [13] detail a load balancing approach in which the node serving a smaller number of users delivers more traffic. It manages to maintain the QoS required by the already established UEs while the number of UEs in the network increases. In turn, a scheme that aims at minimizing the delay of the complete transmission is introduced in [14]. For this purpose, lower traffic flow is delivered by the node that involves a greater delay.

In addition, an appropriate selection of the eNB and gNB that serve each UE allows to maximize this improvement achieved by managing the resources of the suitable nodes. In this regard, the authors of [15] calculate the combination of nodes that maximizes the sum rate capacity. It is computed by using the SINR reported to each node by the UE. However, this solution is proposed for scenarios with a low number of users and nodes since it uses a bruteforce algorithm, so it can trigger the number of different combinations in denser environments. In a similar way, an iterative algorithm that selects two nodes, one from a macro-cell and one from a small-cell, is proposed in [16]. It also uses the SINR to maximize the throughput obtained by UEs. On the other hand, the authors of [17] propose to dynamically add or remove the nodes which simultaneously serve the UE based on Channel State Information (CSI) and cell loads. This scheme aims at increasing the data rate, as well as decreasing the probability of radio link failure. Including the use of the UE location, a deep learning-based mobility management methodology is introduced in [18] that enables dual connection to improve the quality of service of UEs that are going to perform a handover. To this end, the methodology forecasts the future location of users based on mobility patterns. Besides the selection of nodes from two 3GPP access technologies, other studies propose the use of a wireless local area network access point (WAP) to serve the UE in heterogeneous networks [19]. Therefore, both licensed and unlicensed spectrum is used in resource allocation. In this manner, the authors of [20] provide a methodology that dynamically balances the load between eNBs and WAPs. On the other hand, the nodes of these two radio access technologies (RATs) that provide the highest estimated QoE to video streaming users are selected by the approach introduced in [21].

In spite of these solutions, 3GPP proposes to select the nodes based only on Reference Signal Received Power (RSRP) [22]. Given the simplicity of this solution, this paper proposes an alternative methodology to outperform 3GPP approach. The proposed system uses the location of the UEs as well as multiple network metrics to select the eNB and gNB that will provide the maximum QoE to users. In this context, this paper proposes a location-aware solution for the selection of eNB and gNB that simultaneously serve an user in MR-DC-enabled scenarios. A system-level simulation tool is used to compare the proposed methodology and the 3GPP-compliant solution in a real dense urban scenario. In this regard, several tests are carried out with different scenario conditions to demonstrate the feasibility of the proposed solution, which enhance the overall QoE perceived by users. The results also demonstrate the importance of location in properly assigning network nodes in MR-DC scenarios.

The rest of the paper is organized as follows. Section 2 presents the scenario. The methodology proposed is detailed in Section 3 followed by the simulation assumptions in Section 4. The results are then depicted in Section 5. Finally, concluding remarks and future work are outlined in Section 6. 


\section{Scenario}

Several services provided over cellular networks are increasingly demanding more bandwidth. To address this issue, in addition to the use of new features introduced in current non-standalone networks such as MR-DC or millimeter-waves, network operators are deploying network topologies with higher spectrum reuse [23]. Hence, the node intersite distance and transmit power are decreased to set up denser scenarios, which allows to increase the reuse of the same frequencies. This topology is defined by 3GPP as dense urban scenarios, which proposes $200 \mathrm{~m}$ as average inter-site distance [24].

These scenarios can encompass many types of areas with different characteristics, such as user density, predominant services, obstacles distribution, eventuality of social events, etc. For example, residential areas will have the most indoor users, while rural areas will have a low density of users. Moreover, video services will be more frequently used in a residential zone than in a park. There will also be spots with high frequency of social or sporting events, etc. Therefore, in addition to network performance indicators, the characteristics of the environment surrounding the nodes and users must be considered to properly manage network resources.

Furthermore, the service of the UE must be taken into account to maximize his perceived QoE, since each service can have different requirements. This perceived QoE is usually measured using the mean opinion score $(M O S)$, which ranges from 1 (poor experience) to 5 (excellent experience) $[25,26]$. Since the availability of QoE data reported by actual users is often scarce, the MOS is computed using utility functions mapping high-level service performance indicators, e.g., initial playback time for video streaming services [27]. Therefore, different QoS parameters, such as latency or reliability, are intrinsically involved in estimating users' level of satisfaction depending on the type of service [28,29]. Hence, the UE location and a different QoE model for each service are used to maximize the MOS provided by the methodology introduced in the next Section 3.

\section{Node Management Methodology}

The proposed technique selects the eNB and gNB that will serve each user with the highest possible MOS in MR-DC-enabled scenarios. To this end, a set of indicators is used as input of the system. They are detailed as follows:

- $\quad$ Service: The service used by the UE. In this regard, the selection could vary depending on the requirements of the service, i.e., the importance of the throughput, communication reliability, delay, etc. to perceive a good QoE. Thus, the system will use a different configuration for each service.

- Location: The location of the UE. In this sense, the configuration of the system could also change depending on the characteristics of the area that surrounds the UE. In this study, the network operator is assumed to be aware of the UE position by trilateration [30]. This process computes the intersection between circles created between the UE and different network nodes taking advantage of the received signal strength.

- $\quad$ Reference Signal Received Power (RSRP): The RSRP of each candidate eNB and gNB reported by the UE. Only the nodes that overcome a threshold will be proposed as candidates.

- $\quad$ Reference Signal Received Quality (RSRQ): The RSRQ of each candidate eNB and gNB reported by the UE. It may not correlate with the RSRP in some cases, such as when both the received power and interference level are high.

- Available Physical Resource Blocks (PRB): The number of free PRBs in each candidate eNB and gNB. It may rely on the density of users as well as the average bandwidth assigned to each user, which can also depend on the predominant service.

As introduced above, it is important to know the characteristics of the area that surrounds the user before assigning the network nodes. This methodology proposes to divide the scenario into $M * N$ squares, whose surface is assumed to have the same characteristics. The experiences of historical users of each square are used to forecast the MOS that can be provided by each node. With this objective, the RSRP and RSRQ 
reported, as well as the resource availability of the eNB and the gNB that simultaneously served these past users, are proposed to estimate the MOS. Therefore, as each metric could have a different weight to obtain a good QoE for different squares or services, $M * N * S$ estimation models are generated from the introduced metrics to estimate the expected MOS for each service in each square, where $S$ is the number of different services. For instance, the weight of the resource availability could be higher in ultra-dense areas. On the other hand, RSRQ could be more relevant for video users demanding high throughput, while RSRP could be more important for lightweight communications with Internet-of-Things (IoT) sensors. Hence the importance of having different estimation models. Once they are generated and deployed to assign the serving nodes in real time, the network operator will select the corresponding model based on user location and service. It will then take the measurements reported by the UE and the resource availability of each candidate $\mathrm{eNB}$ and gNB to assign the nodes that maximize the MOS and use them as inputs for the following algorithm.

To decrease the computing cost, only the nodes that provide a RSRP over a threshold will be considered as candidates. Moreover, a lightweight technique is used to generate the estimation models: multiple regression technique [31], which is detailed in Equation (1):

$$
\left[\begin{array}{c}
Q o E_{1} \\
Q o E_{2} \\
\vdots \\
Q o E_{n}
\end{array}\right]=\left[\begin{array}{cccc}
1 & P R B_{1} & R S R P_{1} & R S R Q_{1} \\
1 & P R B_{2} & R S R P_{2} & R S R Q_{2} \\
\vdots & \vdots & \vdots & \vdots \\
1 & P R B_{n} & R S R P_{n} & R S R Q_{n}
\end{array}\right]\left[\begin{array}{c}
C \\
W_{\text {Load }} \\
W_{\text {Power }} \\
W_{\text {Quality }}
\end{array}\right]+\left[\begin{array}{c}
\text { Error }_{1} \\
\text { Error }_{2} \\
\vdots \\
\text { Error }_{n}
\end{array}\right]
$$

where $n$ represents the number of historical samples of the same service in a single square from a dataset collected by testing the 3GPP solution in the same scenario. On the other hand, $Q o E_{i}$ indicates the MOS estimated for the UE $i$ by using network Key Quality Indicators (KQIs) as detailed in [32]. In addition, $P R B_{i}, R S R P_{i}$ and $R S R Q_{i}$ represent the number of free PRBs, the signal level and quality metrics, respectively. It should be pointed out that these three measurements represents the mean of the values corresponding to both eNB and $\mathrm{gNB}$, as the MOS value is the result of the service provided by both nodes. Therefore, the estimation model consists of $C$, which is the constant value of the model, and the weights $(W)$ of each introduced metric. In this regard, $C, W_{\text {Load }}, W_{\text {Power }}$ and $W_{\text {Quality }}$ are derived using the historical samples of QoE, $P R B, R S R P$ and $R S R Q$, and the use of an ordinary least squares estimator. This process is detailed in [33]. Afterwards, the QoE will be estimated for future situations as indicated in Equation (2):

$$
E_{t}=C+W_{\text {Load }} * P R B+W_{\text {Power }} * R S R P+W_{\text {Quality }} * R S R Q
$$

where $E_{t}$ is the MOS value estimated by the proposed approach. Therefore, the eNB and the gNB that provide the highest $E_{t}$ values will be selected to serve the UE.

In addition, the Mean Absolute Percentage Error (MAPE) metric is computed to know how reliable is the created model. It indicates the difference between the MOS value $R_{t}$ calculated using the QoE equation of the corresponding service introduced in [32] and the $E_{t}$ value estimated by the proposed methodology (Equation (3)),

$$
\operatorname{MAPE}(\%)=\frac{100}{n} \sum_{t=1}^{n}\left|\frac{R_{t}-E_{t}}{R_{t}}\right|
$$

The lower the MAPE, the higher the estimation accuracy. Therefore, the selection of nodes will be made with more reliability as the MAPE decreases, leading to further increase the overall QoE. The block diagram of this node management solution is represented in Figure 1. It should be noted that in this figure EstimationModel ${ }_{2,3,1}$ is selected because the UE uses the first service and is located in the orange square, which corresponds to the second column and third row in this example. Finally, the algorithm of the proposed methodology is detailed in Algorithm 1. 


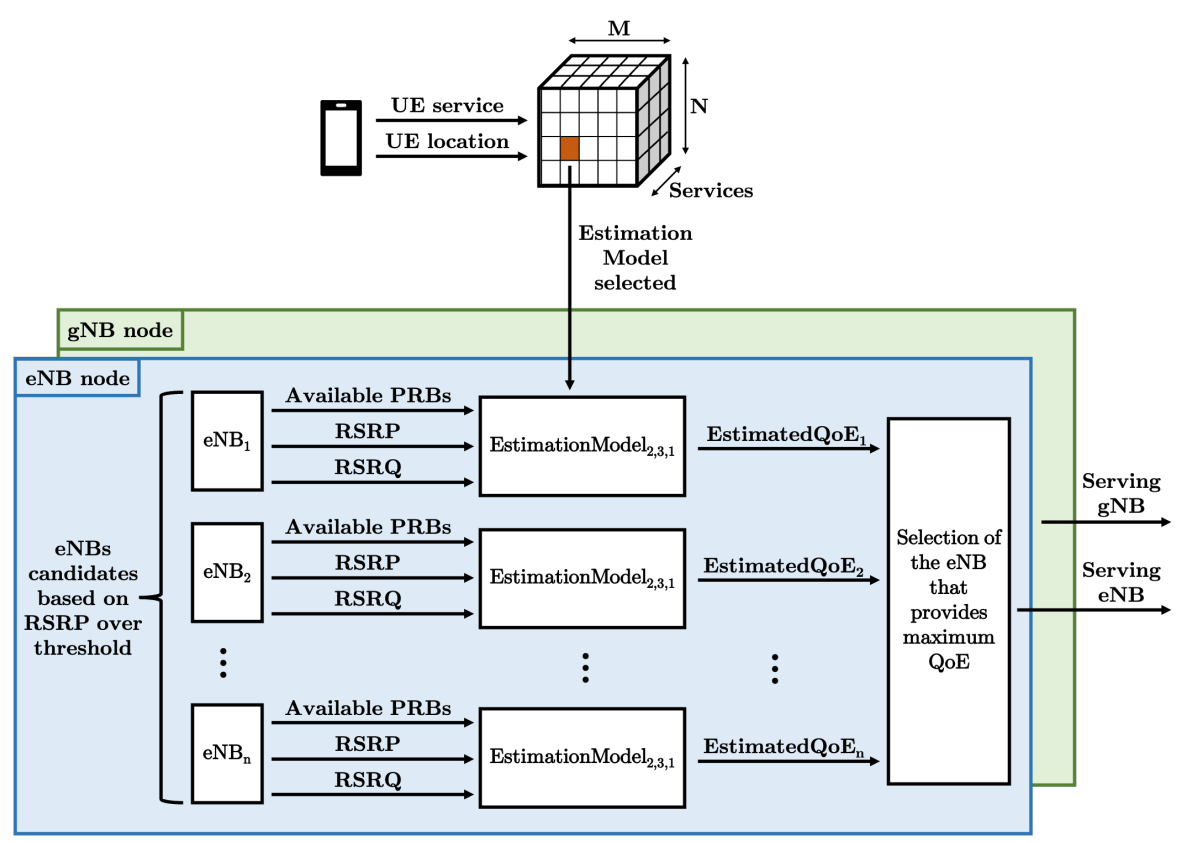

Figure 1. Block diagram of the proposed methodology.

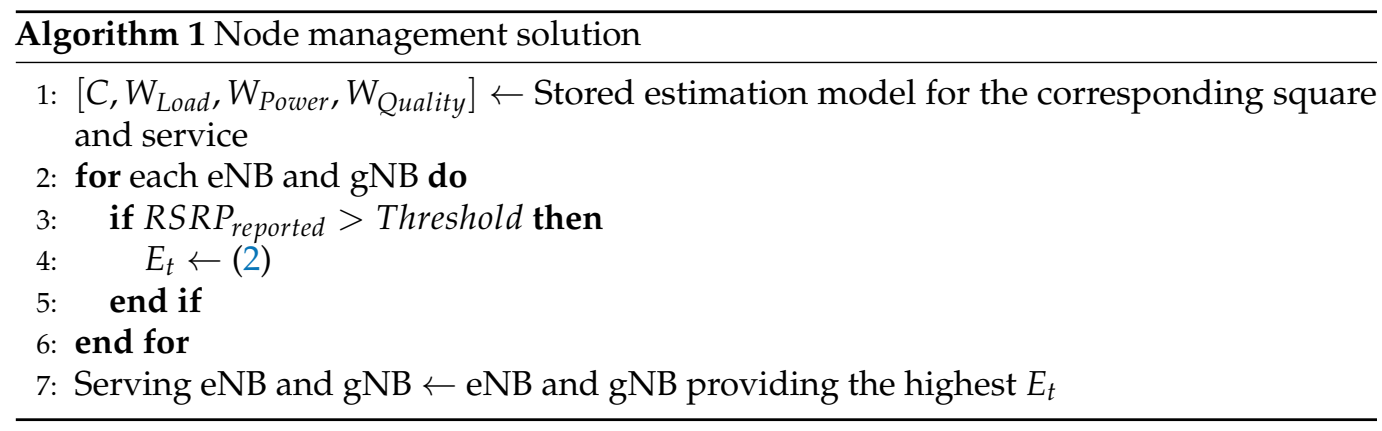

\section{Simulation Assumptions}

The performance of the proposed solution is assessed in a Matlab simulator with MR-DC capabilities, which is based on [34]. The introduced approach is compared with the 3GPP solution based solely on RSRP, so the benefit of the proposed methodology can be highlighted. They are tested in a non-standalone dense urban scenario that correspond to a region of a real celullar network with 20 tri-sectional sites fulfilling 3GPP specification [24]. Figure 2 depicts this scenario. It should be also noted that the eNBs and the gNBs are co-located in each site, so only small-cells are assumed. However, five different scenario setups are proposed to analyze the feasibility of the methodology. In this manner, a uniform distribution of users and services over the whole scenario is set in the first tests, so all regions have equal characteristics. However, the next four scenario setups will consist of four squares of the same size but different characteristics. In particular, an uneven distribution of services is set in the second set of tests. On the other hand, an unequal distribution of users is configured in the third set of tests, resulting in less bandwidth available per UE as density increases. In a similar manner, regions with different level of propagation losses are simulated in the fourth set of tests. Finally, last tests combine the features of the second, third and fourth configurations. Further details of each scenario are collected in Table 1, as well as a graphical representation is shown in Figure 3.

The benefit of the proposed approach is analyzed in terms of MOS reported by users for each of the four services. They are real time video (RTV), video streaming (VS), whose functioning implies a non-real time service, file download (FD) and web browsing (WB) [35]. Their MOS values are computed based on the models introduced in [32]. Regarding FD and WB, it relies on the average session throughput. As for VS service, the MOS depends 
on the initial buffering time as well as the average stalling frequency and duration. Finally, the MOS of RTV relies on the playout time stored in the receiver buffer. Table 2 summarizes the main simulation parameters.

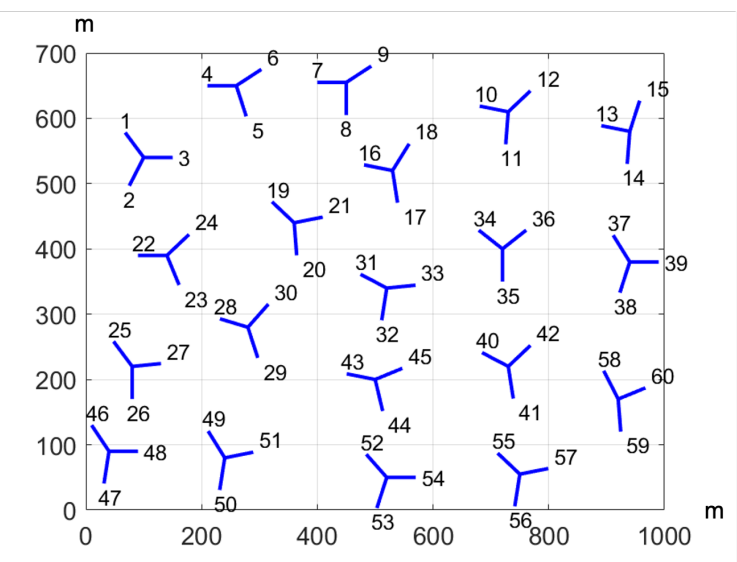

Figure 2. Dense urban scenario considered in this work.

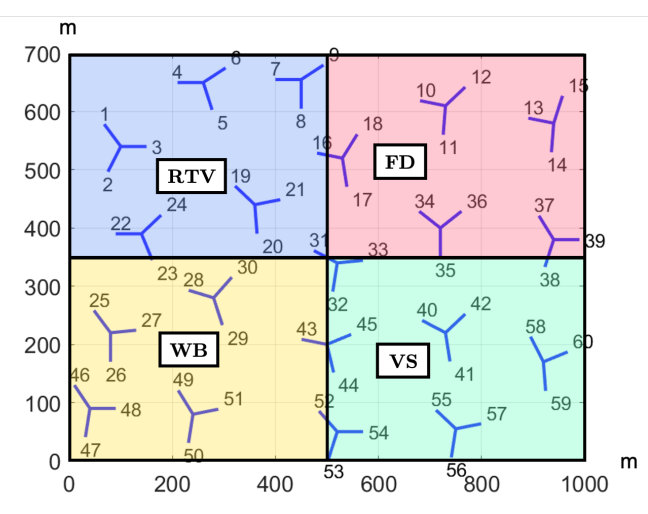

(a)

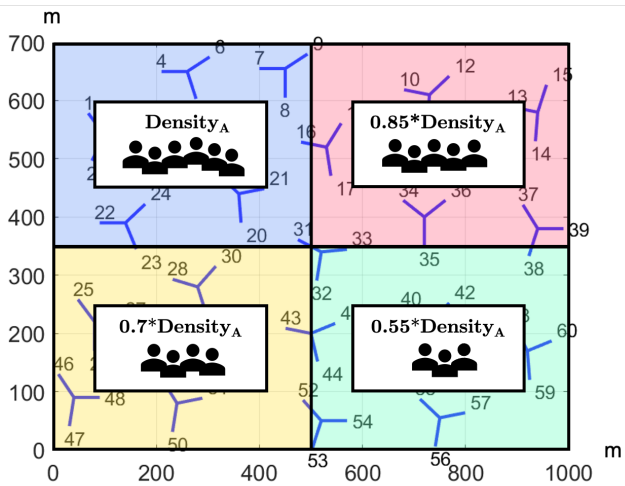

(b)

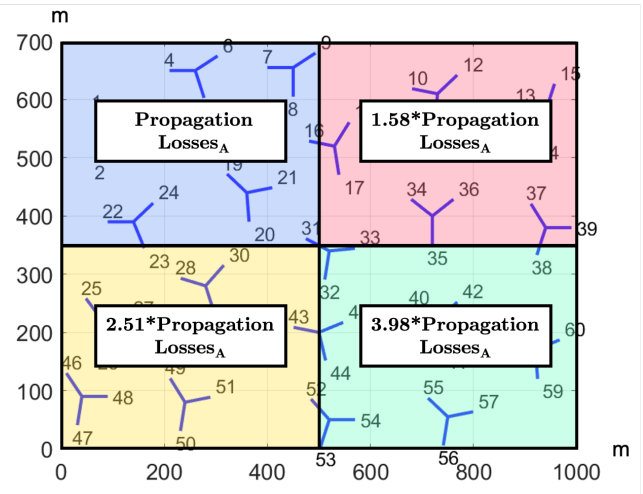

(c)

Figure 3. Configurations of the scenario in second, third and fourth tests. (a) Second scenario setup. (b) Third scenario setup. (c) Fourth scenario setup. 
Table 1. Configuration of the scenario for each test.

\begin{tabular}{|c|c|}
\hline Scenario 1 & $\begin{array}{l}\text { Uniform distribution of users and services } \\
\text { (60 UEs per site and } 300 \text { UEs per service on average) }\end{array}$ \\
\hline Scenario 2 & $\begin{array}{c}\text { Uneven distribution of services } \\
4 \text { squares: } 70 \% \text { of total UEs use the same service in each square } \\
\text { The remaining } 30 \% \text { use the other } 3 \text { services } \\
\text { Predominant service as indicated in Figure } 3 a\end{array}$ \\
\hline Scenario 3 & $\begin{array}{c}\text { Uneven distribution of users } \\
4 \text { squares: Users density decreases by } 15 \% \text { in each square } \\
\text { as indicated in Figure } 3 \mathrm{~b}\end{array}$ \\
\hline Scenario 4 & $\begin{array}{c}\text { Different radio conditions } \\
4 \text { squares: Propagation losses increase in each square } \\
\text { as indicated in Figure } 3 c\end{array}$ \\
\hline Scenario 5 & $\begin{array}{c}4 \text { squares with completely different characteristics } \\
\text { Scenarios } 2,3 \text { and } 4 \text { are combined }\end{array}$ \\
\hline Environment & $\begin{array}{c}\text { Dense urban scenario with } 20 \text { sites, } \\
1 \mathrm{eNB} \text { and } 1 \mathrm{gNB} \text { per site, } 3 \text { sectors } / \text { site }\end{array}$ \\
\hline Carrier & $\begin{array}{l}\text { eNB: } 10 \mathrm{MHz} \text { carrier bandwidth at } 2.4 \mathrm{GHz} \\
\text { gNB: } 10 \mathrm{MHz} \text { carrier bandwidth at } 4 \mathrm{GHz}\end{array}$ \\
\hline Channel model & Urban micro (UMi) [36] \\
\hline PHY numerology & $\begin{array}{c}15 \mathrm{kHz} \text { sub-carrier spacing } \\
12 \text { subcarriers/PRB }(180 \mathrm{kHz})\end{array}$ \\
\hline Data channel MCS & $\begin{array}{c}\text { QPSK to 64 QAM } \\
\text { with same encoding rates as specified for LTE }\end{array}$ \\
\hline Antenna configuration & $2 \times 2 \mathrm{MIMO}$ \\
\hline Scheduler & Classical exponential/proportional fair [37] \\
\hline Link adaptation & CQI-based \\
\hline Mobility model & $50 \%$ chance of the user being static or moving at $0.83 \mathrm{~m} / \mathrm{s}$ \\
\hline FD traffic model & File size: log-normal distribution (avg. $2 \mathrm{MB}$ ) \\
\hline WB traffic model & $\begin{array}{l}\text { Web page size: log-normal distribution (avg. } 3 \mathrm{MB} \text { ) } \\
\text { No. pages per session: log-normal distribution (avg. } 4 \text { ) } \\
\text { Reading time: exponential distribution (avg. } 30 \mathrm{~s})\end{array}$ \\
\hline RTV traffic model & $\begin{array}{c}\text { Video codec: H.264, Resolution: } 720 \mathrm{p} \\
\text { Video bitrate range: } 1.5-2 \mathrm{Mbps} \\
\text { Video duration: uniform distribution }[0,540] \mathrm{s}\end{array}$ \\
\hline VS traffic model & $\begin{array}{l}\text { Video codec: H.264, Resolution: } 1080 \mathrm{p} \\
\text { Video bitrate range: } 3-4 \mathrm{Mbps} \\
\text { Video duration: uniform distribution }[0,540] \mathrm{s}\end{array}$ \\
\hline
\end{tabular}

\section{Evaluation}

First of all, the optimal square size must be defined. This will indicate the number of estimation models to be stored, as well as the required accuracy in locating the users. With this purpose, the MAPE obtained for each service with different square sizes is computed for all the scenario setups introduced in the previous section. Figure 4 shows that the MAPE decreases for all services as the square side size decreases in every scenario, i.e., the scenario is characterized with more estimation models. However, the values are different for each scenario configuration. The lowest overall MAPE values are obtained in scenarios 2 and 5 , as can be seen in the Figure $4 b$,e. This indicates that the MAPE tends to be lower in small regions where there is a predominant service whose performance is well 
known. However, the MAPE of the RTV service is higher in these scenarios because the increase in user density causes an increase in the overall delay that negatively affects the QoE perceived by users and is not directly measured by the proposed approach. On the other hand, the MAPE obtained for the other services is similar in each scenario, as they are more dependent on the throughput, which can be better estimated in small regions where local radio conditions and typical resource availability are more accurately known. In conclusion, the lowest MAPE values are obtained for areas with $20 \mathrm{~m}$ of square side size. Nonetheless, $30 \mathrm{~m}$ of square side size is proposed to generate the estimation models used in the next tests. This decision enables a compromise between the most reliable decisions possible and the minimum memory and computing cost, since the difference between the MAPE obtained for 20 and $30 \mathrm{~m}$ of square side size is relatively small. Moreover, this allows for a larger error in tracking the UE location.

In addition, the importance of each metric in the QoE estimation must be analyzed. In this way, the weight of each metric for each service in all scenarios is represented in a box plot in Figure 5. These values are obtained after normalizing the weight of each metric in each estimation model generated for areas of $30 \mathrm{~m}$ of square side size. Figure $5 \mathrm{a}$ shows that RSRP is the least important metric for QoE estimation, except for the RTV service since RSRP is correlated with the distance between the nodes and the user, which may have an impact on the service latency and, therefore, on the QoE perceived. On the other hand, RSRQ and available PRBs have similar weights for less resource demanding services, i.e., FD and WB. On the contrary, available PRBs are more important for video services, especially for the most resource and throughput demanding service, i.e., VS. In addition, the relevance of available resources will increase in Scenario 2 for all services, as shows in Figure 5b, since the higher density of video users in two different areas will lead to overload these regions. Moreover, resource availability will be more important for WB users which do not demand as much throughput as FD users, which will also rely heavily on RSRQ. Furthermore, RSRQ is also very relevant for video users to perceive a good QoE. On the other hand, the decrease in user density does not affect the weights much, as the values represented in Figure $5 \mathrm{c}$ are similar to those obtained in Scenario 1. Conversely, the increase in propagation losses does affect the RTV service, which is more dependent on RSRP, as depicted in Figure 5d. These additional propagation losses could indicate a longer distance between nodes and users, which is relevant for RTV with strict delay requirements. Finally, Figure 5e shows that available PRBs are considerably more important than the others metrics when there are heavily loaded areas in the scenario, as in Scenario 2. After resource availability, RSRQ is the most important metric. It should also be noted that the relevance of RSRP for RTV users is low in this scenario because there are no additional propagation losses in the area where this service predominates. Therefore, this set of subfigures shows that RSRP, which is the only metric used in the 3GPP solution, is the indicator that has the lowest weight for estimating QoE. This implies that RSRQ and available resources should be used to decide the nodes that will serve an user if MOS is to be maximized. Last but not least, the variance of the values indicates that the weights change throughout the scenario, verifying the requirement of multiple estimation models. 


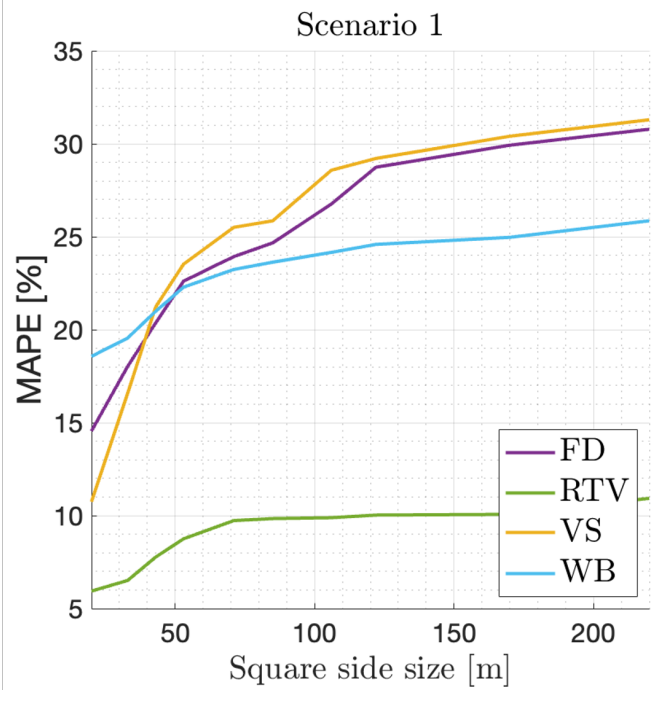

(a)

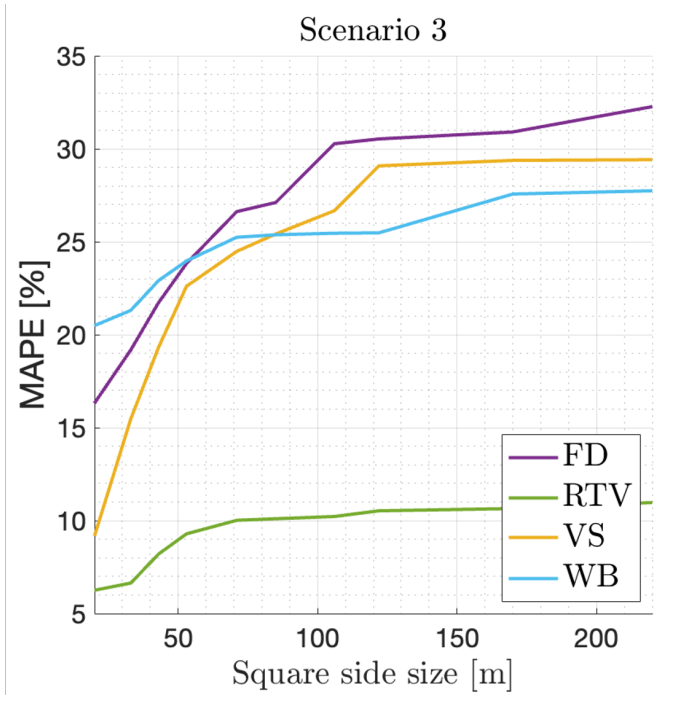

(c)

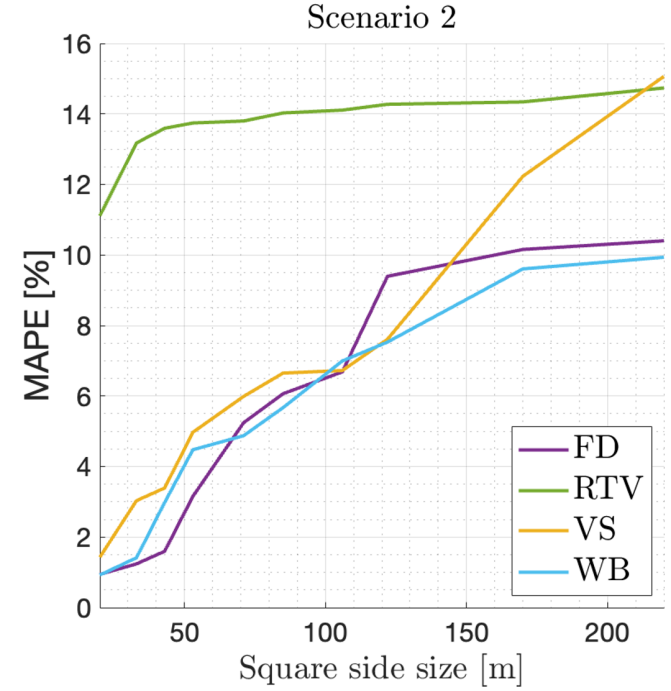

(b)

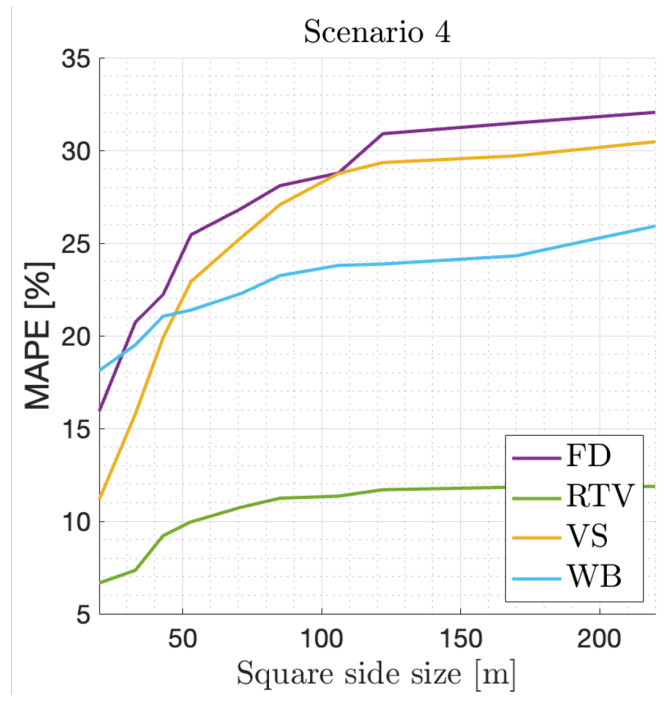

(d)

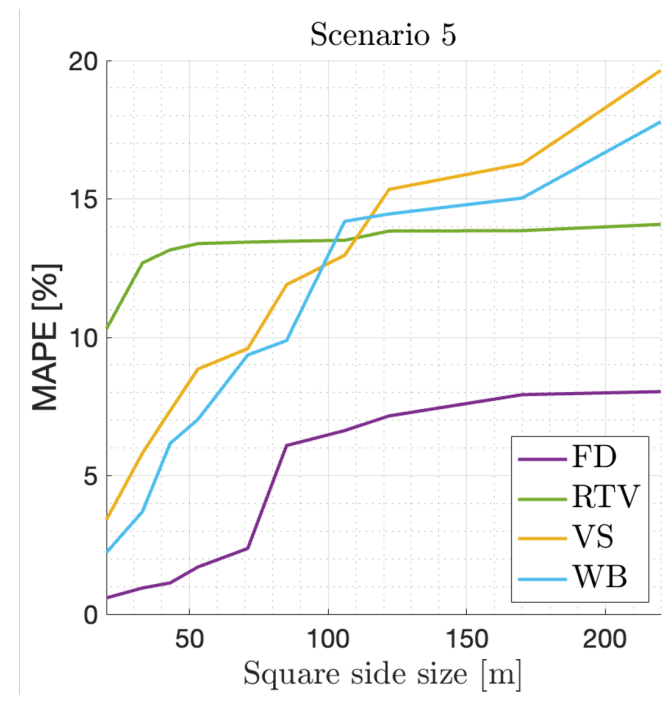

(e)

Figure 4. MAPE for different square side sizes, services and scenarios. (a) MAPE in Scenario 1. (b) MAPE in Scenario 2. (c) MAPE in Scenario 3. (d) MAPE in Scenario 4. (e) MAPE in Scenario 5. 


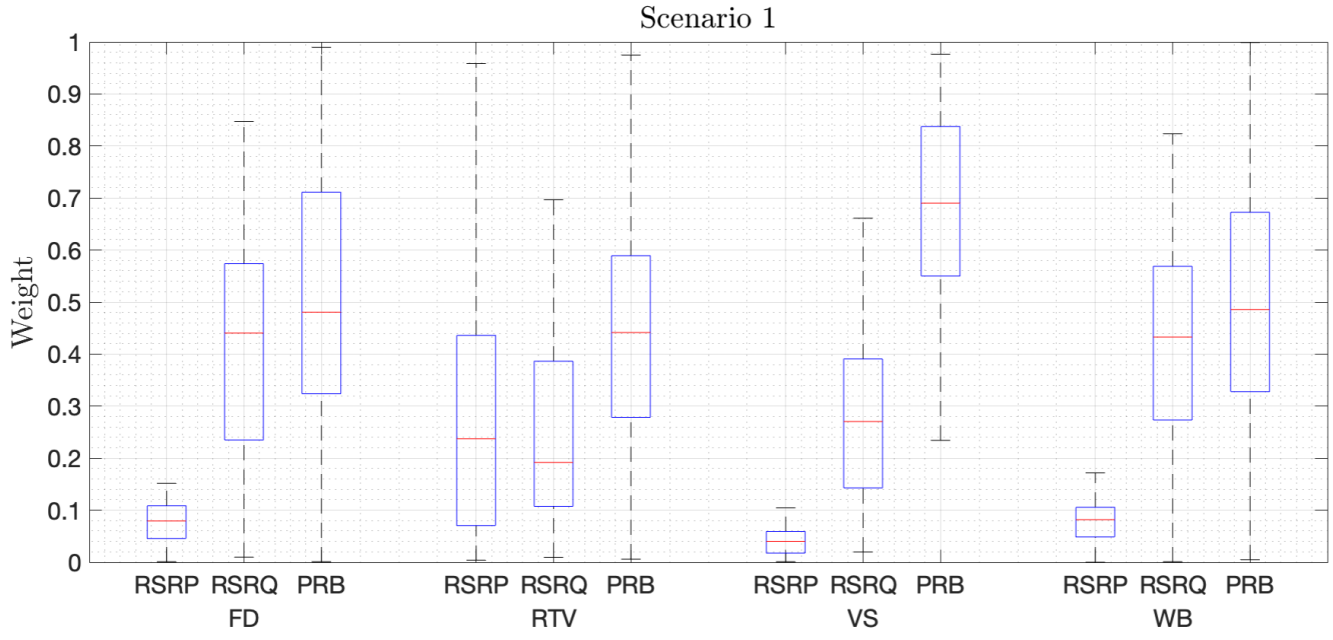

(a)

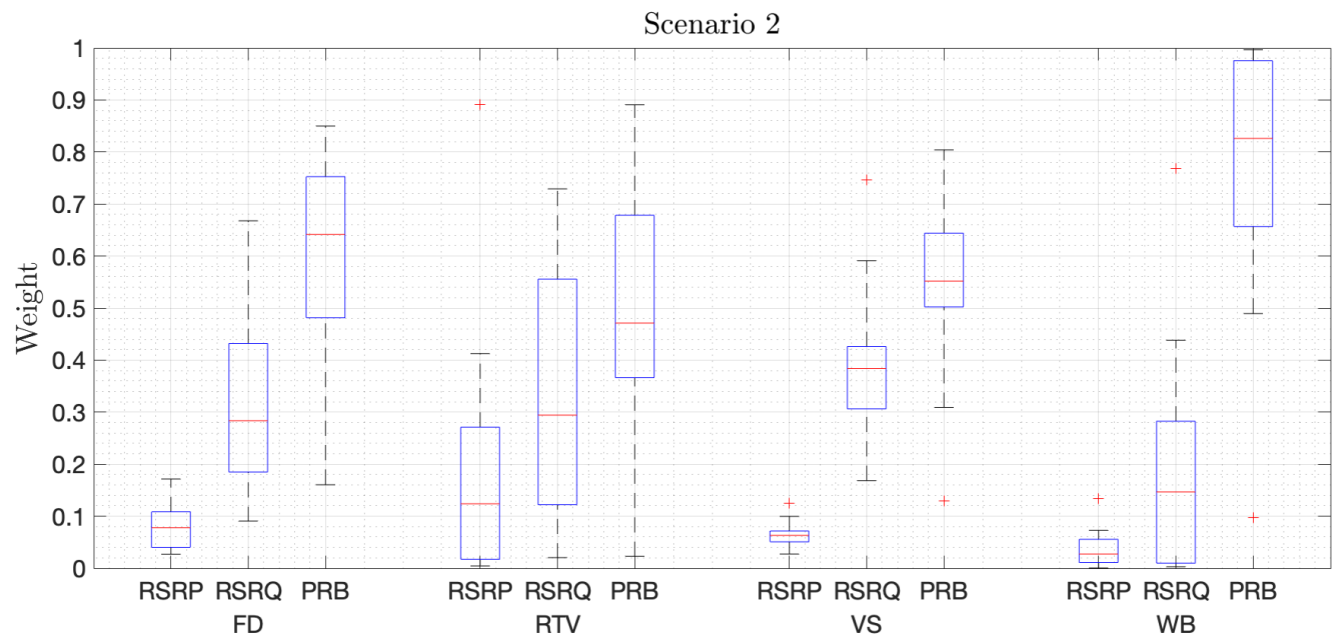

(b)

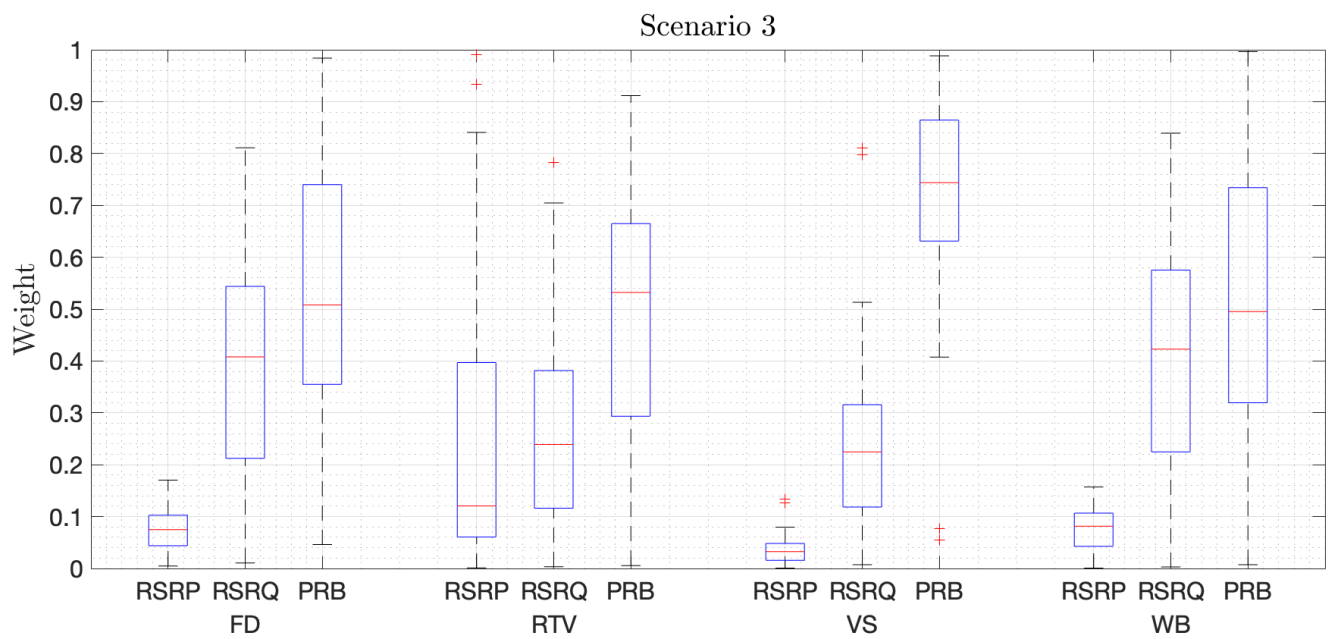

(c)

Figure 5. Cont. 


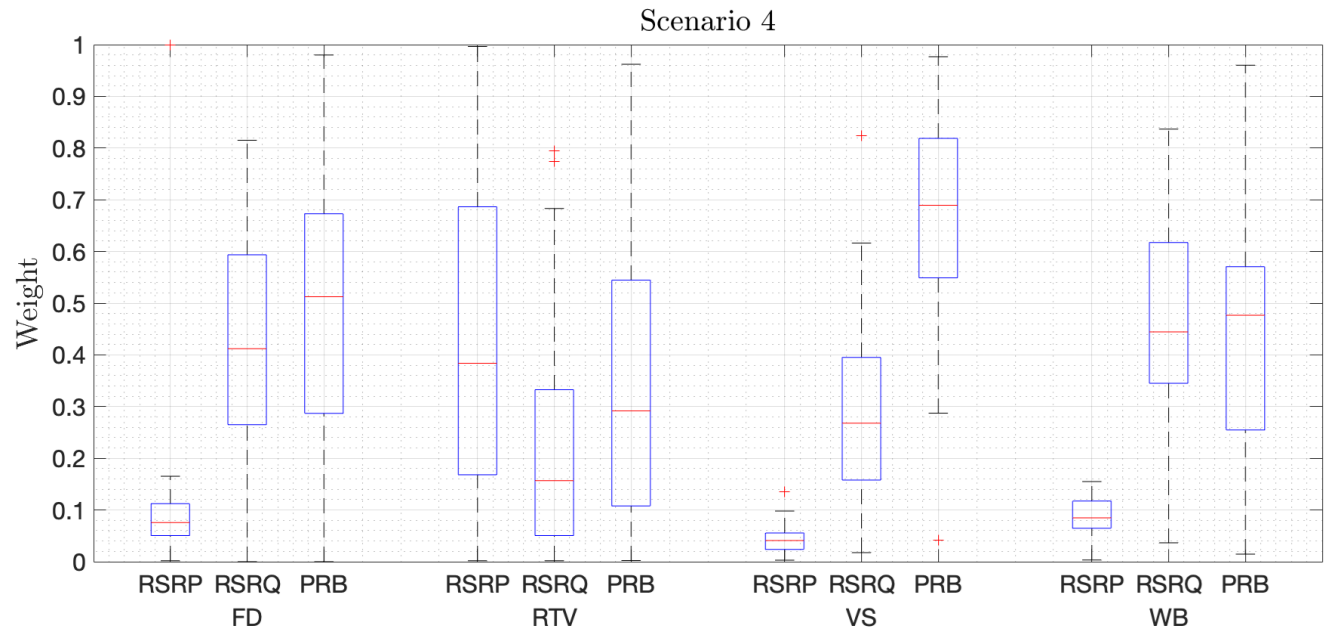

(d)

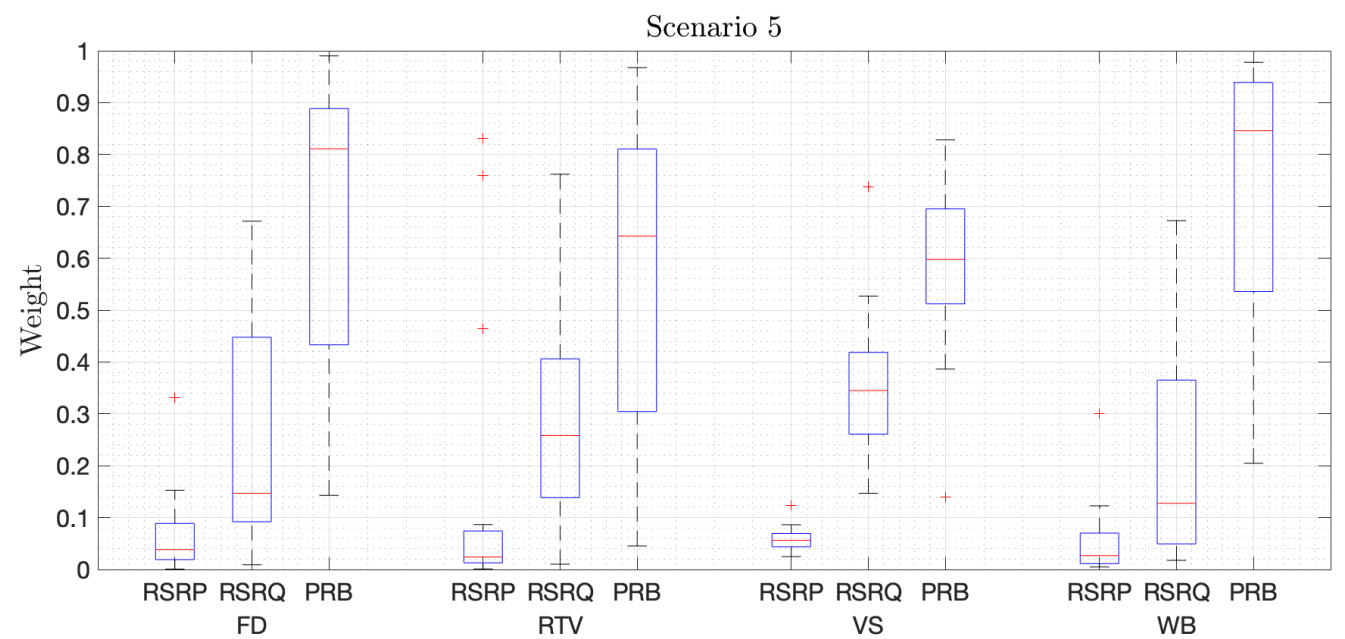

(e)

Figure 5. Weight of each metric of the estimation model for each service and scenario. (a) Weights in Scenario 1. (b) Weights in Scenario 2. (c) Weights in Scenario 3. (d) Weights in Scenario 4. (e) Weights in Scenario 5.

The previously stored estimation models are now used to select the eNB and gNB that provide the maximum estimated QoE in these tests. The resulting performance is compared with that obtained in the same scenarios but selecting the nodes based solely on RSRP, as indicated by 3GPP. Results show that the proposed solution increases the average MOS obtained by the 3GPP approach for all the services introduced in every scenario, as depicted in Figure 6. Nevertheless, the average MOS achieved varies depending on the scenario configuration. Figure $6 \mathrm{~b}$ shows how the average MOS of least demanding services increases in the areas where they are predominant with respect to that obtained in the uniform scenario shown in Figure 6a. On the contrary, the MOS of video services decreases due to the high struggle for radio resources in regions where video services are predominant. On the other hand, the MOS of all services increases in Scenario 3 as the user density decreases (Figure 6c). In contrast, the opposite effect is observed when propagation losses grow (Figure 6d). To conclude, Figure 6e shows that the MOS of the predominant services in regions with lower user density is larger despite higher propagation losses. However, the QoE perceived by RTV users is similar to that perceived in Scenario 2, as most RTV users have similar conditions in these two scenarios, as can be seen in Figure 3. 

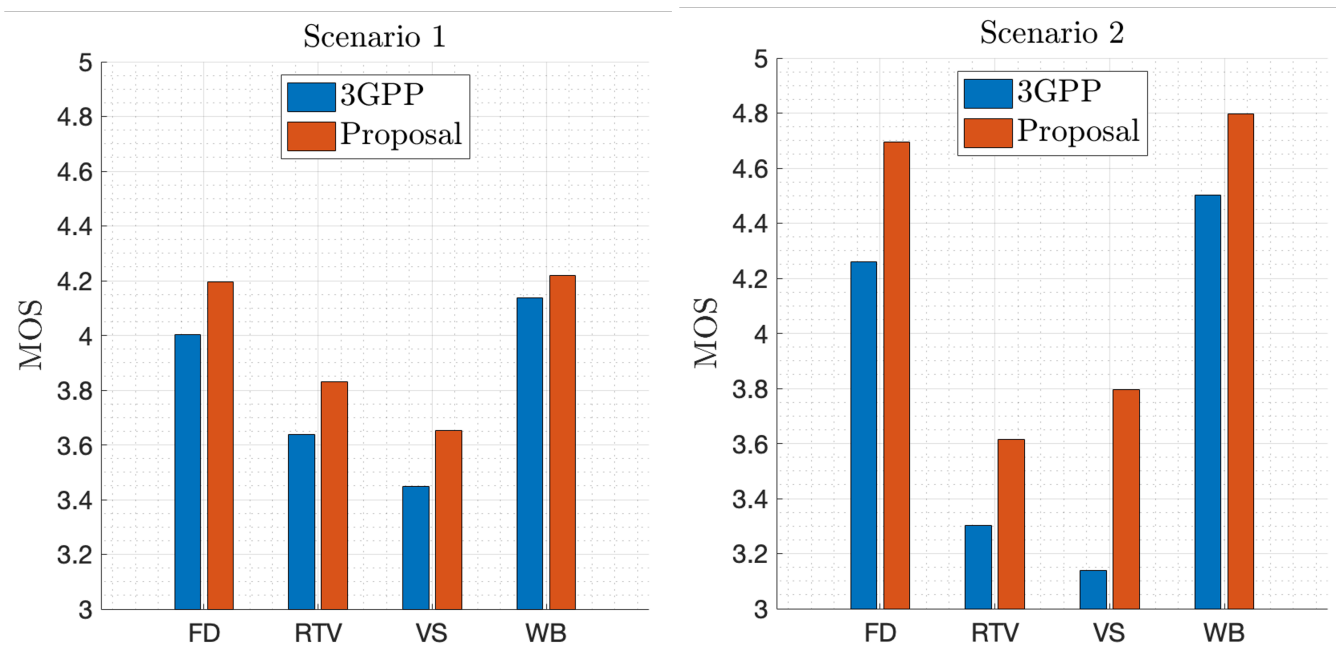

(a)

(b)
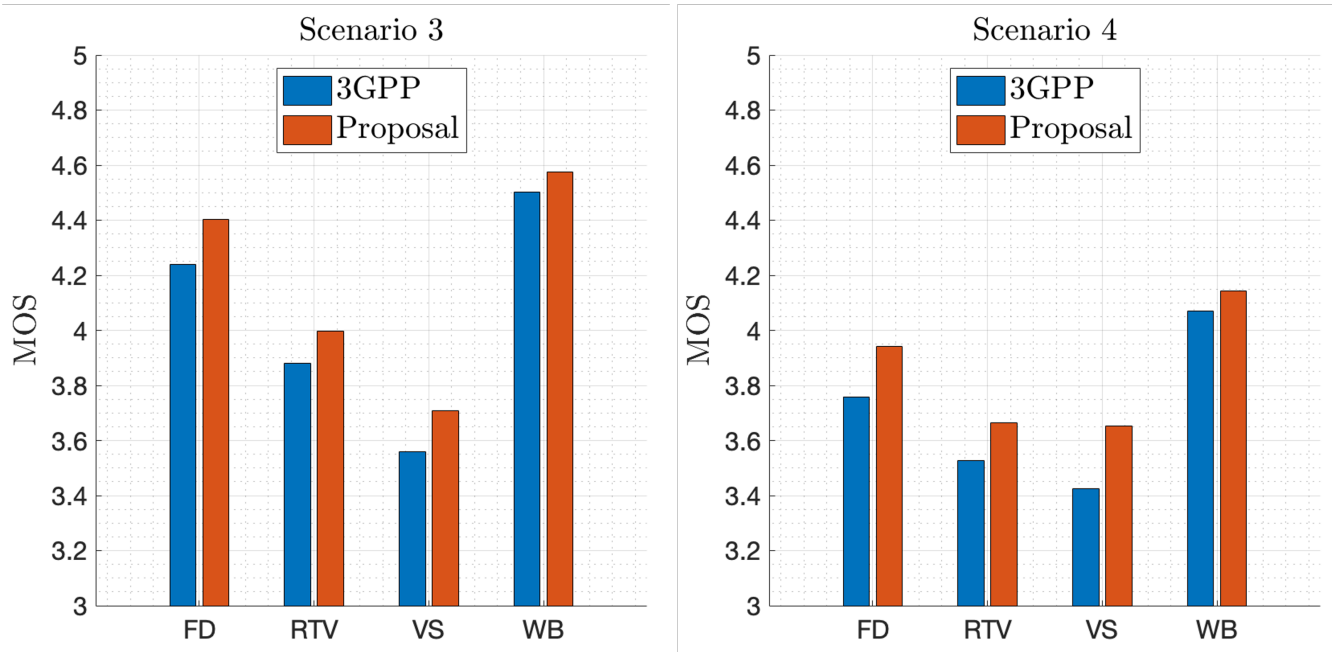

(c)

(d)

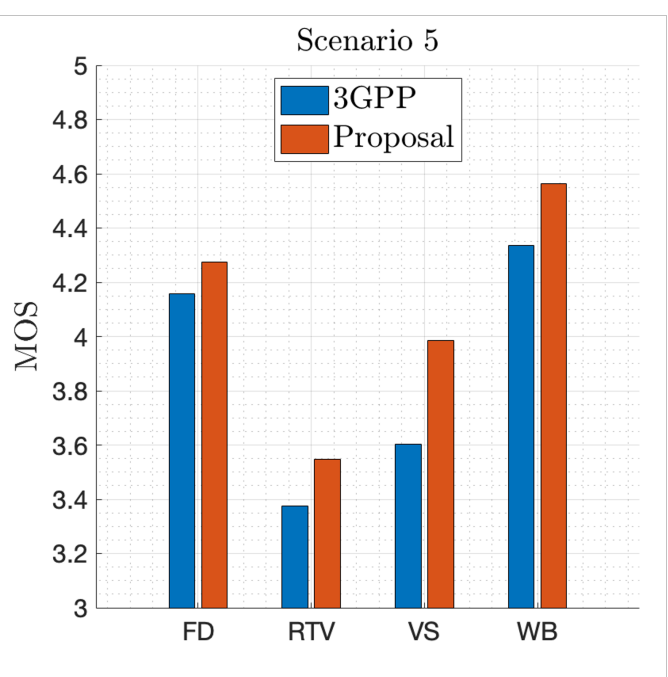

(e)

Figure 6. MOS obtained by each solution for each service in every scenario. (a) MOS in Scenario 1. (b) MOS in Scenario 2. (c) MOS in Scenario 3. (d) MOS in Scenario 4. (e) MOS in Scenario 5. 
To thoroughly analyze the improvement in each service and scenario, Figure 7 represents the percentage of gain of the proposed methodology compared to the 3GPP solution. As can be noted, WB almost always obtains the lowest gain because these users are the least demanding and already perceive a good QoE when using MR-DC, so the MOS is not much affected by the proposed node selection. However, FD users demand more throughput. Therefore, the higher QoE gain indicates that this throughput improvement is more valued by FD users. On the other hand, the MOS of video users is lower than that of FD and WB users in all scenarios (Figure 6), as they demand higher throughput as well as greater availability of radio resources that does not lead to video stalls. In this sense, the MOS of VS users is even lower due to the higher video quality. Hence, the MOS gain obtained by proper node management is more noticeable for this service, especially in scenarios 2 and 5 where there are regions with a large number of VS users struggling to access network resources. To conclude, it is also worth noting that the MOS improvement is more prominent in Scenario 2 (Figure $7 \mathrm{~b}$ ), where different regions of the network usually have a high traffic flow of the same service, making it particularly fruitful to deploy the proposed approach in this type of regions. Therefore, the results demonstrate that the QoE perceived by users of a mobile network can be optimized by an alternative node management in MR-DC-enabled scenarios. To maximize the QoE, the methodology must adapt to the characteristics of the area surrounding the users as well as the service used. In addition, network load and quality metrics must be considered.
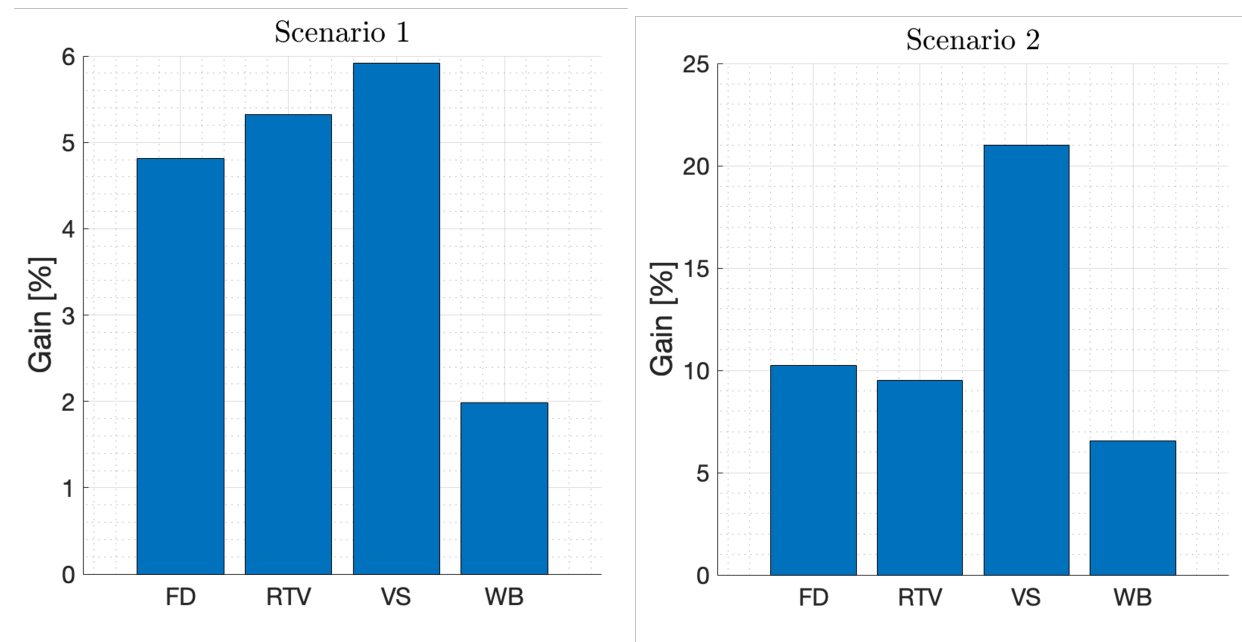

(a)

(b)
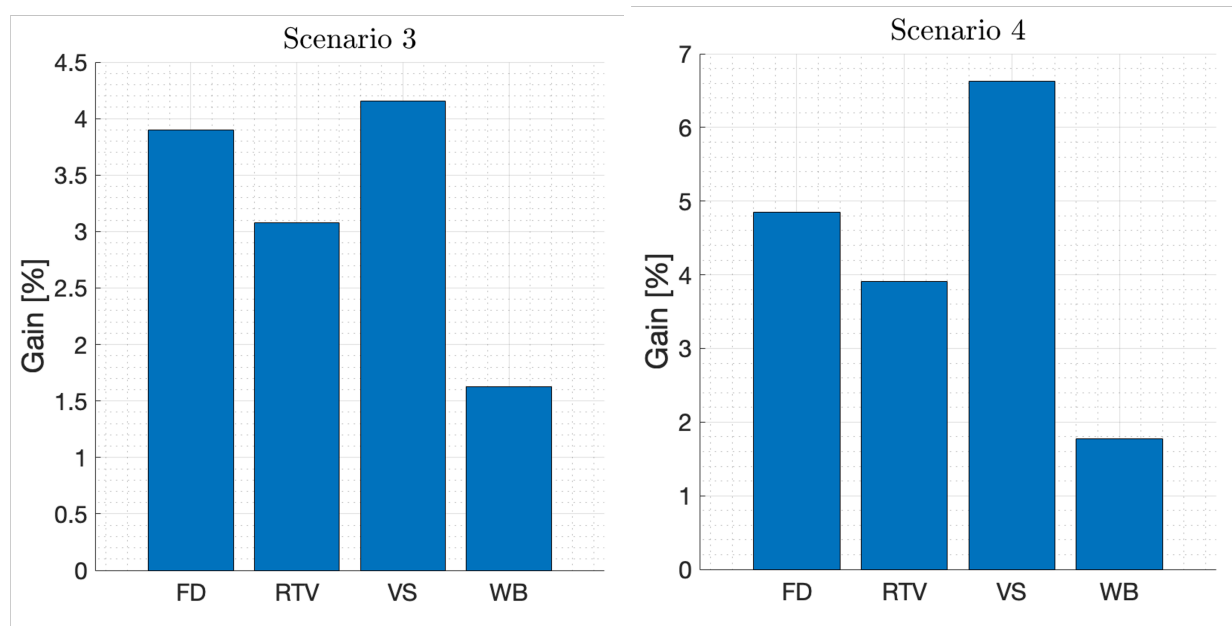

(c)

(d)

Figure 7. Cont. 


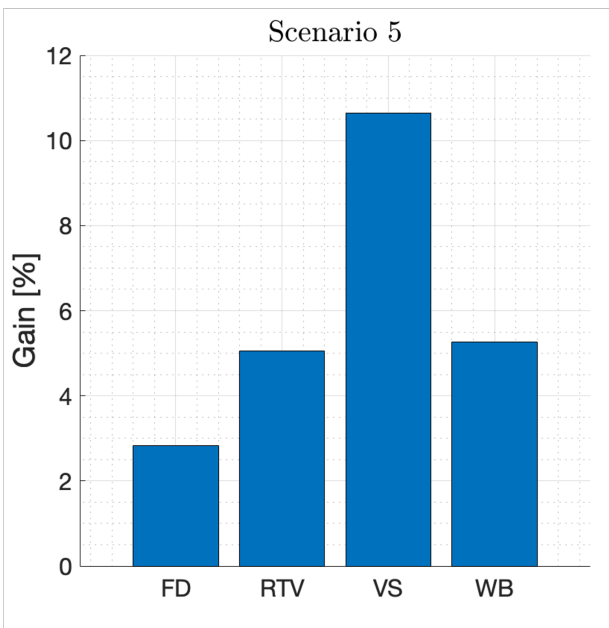

(e)

Figure 7. Percentage of gain achieved by the proposed solution for each service in every scenario. (a) Gain in Scenario 1. (b) Gain in Scenario 2. (c) Gain in Scenario 3. (d) Gain in Scenario 4. (e) Gain in Scenario 5.

\section{Conclusions and Further Work}

The arrival of MR-DC enables great flexibility in the management of network resources, since two nodes are simultaneously connected to a user. Among other advantages, this allows to increase the bandwidth allocated to each user, which can have a positive impact on the QoE. However, a more complex resource management must be tackled. The proposed methodology assigns the user the eNB and gNB that will provide the highest QoE. The results show how this decision varies depending on the characteristics of the area surrounding the user, in addition to the service used. Furthermore, the results demonstrate how resource availability and user-reported RSRQ are more relevant than RSRP in making this selection. In this regard, the performance tests show the improvement of the average QoE compared to the 3GPP solution in a wide variety of scenarios.

The methodology has been tested with different services used by indoor and pedestrian users, but other services involving faster mobility can lead to complications in the dynamic management of connections. Hence, an additional subsystem must be added to address these challenges. This study is left for future work.

Author Contributions: Conceptualization, J.B. and I.d.-1.-B.; methodology, J.B.; software, J.B. and I.d.-1.-B.; validation, J.B.; formal analysis, J.B.; investigation, J.B.; resources, J.B. and I.d.-1.-B.; data curation, J.B.; writing-original draft preparation, J.B.; writing-review and editing, I.d.-1.-B. and R.B.; visualization, J.B., I.d.-1.-B. and R.B.; supervision, I.d.-1.-B. and R.B.; project administration, R.B.; funding acquisition, R.B. All authors have read and agreed to the published version of the manuscript.

Funding: This research was performed in the framework of the Horizon 2020 project LOCUS (grant agreement n. 871249), receiving funds from the European Union.

Institutional Review Board Statement: Not applicable.

Informed Consent Statement: Not applicable.

Data Availability Statement: Not applicable.

Conflicts of Interest: The authors declare no conflict of interest. The funders had no role in the design of the study; in the collection, analyses, or interpretation of data; in the writing of the manuscript, or in the decision to publish the results. 


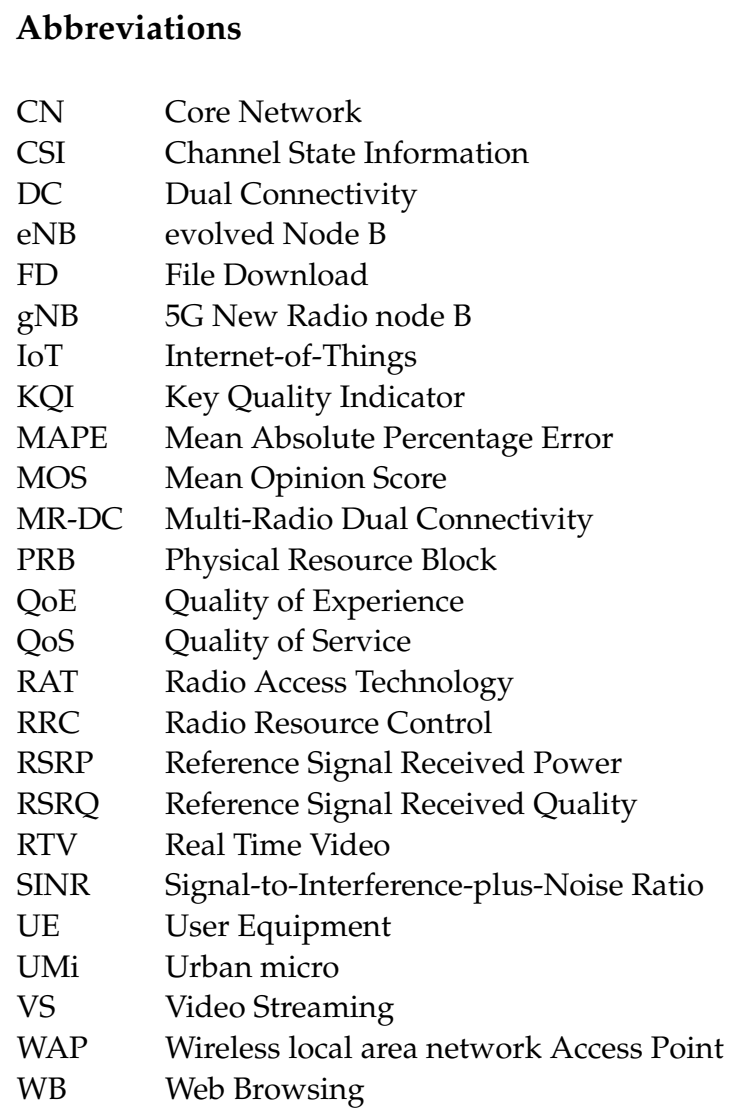

\section{References}

1. Lee, C.-Y.; Lee, C.F.; Tsai, H.-M. An economic assistance strategy for autonomous driving system. In Proceedings of the 2015 International Conference on Advanced Robotics and Intelligent Systems (ARIS), Taipei, Taiwan, 29-31 May 2015; pp. 1-4. [CrossRef]

2. Naik, P.; Desai, P.V.; Pati, S. Location Based Place Recommendation using Social Network. In Proceedings of the 2019 IEEE 5th International Conference for Convergence in Technology (I2CT), Bombay, India, 29-31 March 2019; pp. 1-5. [CrossRef]

3. Fortes, S.; Baena, C.; Villegas, J.; Baena, E.; Asghar, M.Z.; Barco, R. Location-Awareness for Failure Management in Cellular Networks: An Integrated Approach. Sensors 2021, 21, 1501. [CrossRef] [PubMed]

4. Choi, J.; Shin, D. Location-Aware Self-Optimization for Interference Management in Ultra-Dense Small Cell Networks. IEEE Commun. Lett. 2018, 22, 2555-2558. [CrossRef]

5. Jeong, M.; Orikumhi, I.; Kim, S. Location-aware Power Adaptation Scheme for UAV Communications. In Proceedings of the TENCON 2018-2018 IEEE Region 10 Conference, Jeju Island, Korea, 28-31 October 2018; pp. 1624-1627. [CrossRef]

6. Monserrat, J.F.; Bouchmal, F.; Martin-Sacristan, D.; Carrasco, O. Multi-Radio Dual Connectivity for 5G Small Cells Interworking. IEEE Commun. Stand. Mag. 2020, 4, 30-36. [CrossRef]

7. Fokin, G. Interference Suppression Using Location Aware Beamforming in 5G Ultra-Dense Networks. In Proceedings of the 2020 IEEE Microwave Theory and Techniques in Wireless Communications (MTTW), Riga, Latvia, 1-2 October 2020; pp. 13-17. [CrossRef]

8. 3GPP. TS 37.340, NR; Multi-Connectivity; Overall Description; Stage-2; V15.7.0; Rel-15. Available online: https:/ / portal.3gpp. org/desktopmodules/Specifications/SpecificationDetails.aspx?specificationId=3198 (accessed on 2 May 2021).

9. Mahmood, N.H.; Lopez, M.; Laselva, D.; Pedersen, K.; Berardinelli, G. Reliability Oriented Dual Connectivity for URLLC services in 5G New Radio. In Proceedings of the 2018 15th International Symposium on Wireless Communication Systems (ISWCS), Lisbon, Portugal, 28-31 August 2018; pp. 1-6. [CrossRef]

10. Centenaro, M.; Laselva, D.; Steiner, J.; Pedersen, K.; Mogensen, P. System-Level Study of Data Duplication Enhancements for 5G Downlink URLLC. IEEE Access 2020, 8, 565-578. [CrossRef]

11. Guo, Y.; Meng, Q.; Wang, X. Energy-Optimized 5G Dual Connectivity Radio Resource Allocation. In Proceedings of the 2019 IEEE 2nd International Conference on Electronics Technology (ICET), Chengdu, China, 10-13 May 2019; pp. 126-130. [CrossRef]

12. Wu, Y.; Qian, L.P.; Zheng, J.; Zhou, H.; Shen, X.S. Green-Oriented Traffic Offloading through Dual Connectivity in Future Heterogeneous Small Cell Networks. IEEE Commun. Mag. 2018, 56, 140-147. [CrossRef]

13. Simsek, M.; Hößler, T.; Jorswieck, E.; Klessig, H.; Fettweis, G. Multiconnectivity in Multicellular, Multiuser Systems: A MatchingBased Approach. Proc. IEEE 2019, 107, 394-413. [CrossRef] 
14. Zhang, B.; Xu, X.; Zhang, K.; Zhang, J.; Guan, H.; Zhang, Y.; Zhang, Y.; Zheng, N.; Teng, Y. Goodput-Aware Traffic Splitting Scheme with Non-ideal Backhaul for 5G-LTE Multi-Connectivity. In Proceedings of the 2019 IEEE Wireless Communications and Networking Conference (WCNC), Marrakesh, Morocco, 15-18 April 2019; pp. 1-6. [CrossRef]

15. Kim, M.; Jung, S.Y.; Kim, S. Sum-rate maximizing cell association via dual-connectivity. In Proceedings of the 2015 International Conference on Computer, Information and Telecommunication Systems (CITS), Gijón, Spain, 15-17 July 2015; pp. 1-5. [CrossRef]

16. Moein, E.; Taskou, S.K.; Rasti, M. Base Station Assignment in Two-tier Dual Connectivity Heterogeneous Networks. In Proceedings of the 2018 9th International Symposium on Telecommunications (IST), Tehran, Iran, 17-19 September 2018; pp. 474-480. [CrossRef]

17. Ba, X.; Wang, Y. Load-Aware Cell Select Scheme for Multi-Connectivity in Intra-Frequency 5G Ultra Dense Network. IEEE Commun. Lett. 2019, 23, 354-357. [CrossRef]

18. Wang, C.; Zhao, Z.; Sun, Q.; Zhang, H. Deep Learning-Based Intelligent Dual Connectivity for Mobility Management in Dense Network. In Proceedings of the 2018 IEEE 88th Vehicular Technology Conference (VTC-Fall), Chicago, IL, USA, 27-30 August 2018; pp. 1-5. [CrossRef]

19. Anedda, M.; Muntean, G.; Murroni, M. Adaptive real-time multi-user access network selection algorithm for load-balancing over heterogeneous wireless networks. In Proceedings of the 2016 IEEE International Symposium on Broadband Multimedia Systems and Broadcasting (BMSB), Nara, Japan, 1-3 June 2016; pp. 1-4. [CrossRef]

20. Anedda, M.; Murroni, M.; Muntean, G. A Novel Markov Decision Process-Based Solution for Improved Quality Prioritized Video Delivery. IEEE Trans. Netw. Serv. Manag. 2020, 17, 592-606. [CrossRef]

21. Bouali, F.; Moessner, K.; Fitch, M. A context-aware QoE-driven strategy for adaptive video streaming in 5G multi-RAT environments. In Proceedings of the 2017 20th International Symposium on Wireless Personal Multimedia Communications (WPMC), Bali, Indonesia, 17-20 December 2017; pp. 354-360. [CrossRef]

22. 3GPP. TR 36.842, Study on Small Cell Enhancements for E-UTRA and E-UTRAN; Higher Layer Aspects; Rel-12. Available online: https:/ / portal.3gpp.org/desktopmodules/Specifications/SpecificationDetails.aspx?specificationId=2543 (accessed on 2 May 2021).

23. Andrews, J.G.; Buzzi, S.; Choi, W.; Hanly, S.V.; Lozano, A.; Soong, A.C.K.; Zhang, J.C. What Will 5G Be? IEEE J. Sel. Areas Commun. 2014, 32, 1065-1082. [CrossRef]

24. 3GPP. TR 38.913. Study on Scenarios and Requirements for Next Generation Access Technologies; Rel-15. Available online: https:/ / portal.3gpp.org/desktopmodules/Specifications/SpecificationDetails.aspx?specificationId=2996 (accessed on 2 May 2021).

25. Altozano, M.L.M.; Toril, M.; Luna-Ramírez, S.; Gijón, C. A Self-Tuning Algorithm for Optimal QoE-Driven Traffic Steering in LTE. IEEE Access 2020, 8, 156707-156717. 10.1109/ ACCESS.2020.3019281. [CrossRef]

26. Gómez, G.; Lorca, J.; García, R.; Pérez, Q. Towards a QoE-driven resource control in LTE and LTE-A networks. J. Comput. Netw. Commun. 2013. [CrossRef]

27. Gijón, C.; Toril, M.; Luna-Ramírez, S.; Marí-Altozano, M.L. A Data-Driven Traffic Steering Algorithm for Optimizing User Experience in Multi-Tier LTE Networks. IEEE Trans. Veh. Technol. 2019, 68, 9414-9424. [CrossRef]

28. Shahzad, M.; Antoniou, J. Quality of User Experience in 5G-VANET. In Proceedings of the 2019 IEEE 24th International Workshop on Computer Aided Modeling and Design of Communication Links and Networks (CAMAD), Limassol, Cyprus, 11-13 September 2019; pp. 1-6. [CrossRef]

29. Odarchenko, R.; Abakumova, A.; Usik, P.; Smirnov, O.A.; Kundyz, M. QoE Optimization Technique for Media Delivery in 5G Networks. In Proceedings of the 2019 IEEE International Scientific-Practical Conference Problems of Infocommunications, Science and Technology (PIC S\&T), Kiev, Ukraine, 8-11 October 2019; pp. 597-601. [CrossRef]

30. del Peral-Rosado, J.A.; Raulefs, R.; López-Salcedo, J.A.; Seco-Granados, G. Survey of Cellular Mobile Radio Localization Methods: From 1G to 5G. IEEE Commun. Surv. Tutor. 2018, 20, 1124-1148. [CrossRef]

31. Shin, Y.; Chae, C.; Kim, S. Multiple regression models for a big data empowered SON framework. In Proceedings of the 2015 Seventh International Conference on Ubiquitous and Future Networks, Sapporo, Japan, 7-10 July 2015; pp. 982-984. [CrossRef]

32. Burgueño, J.; de-la-Bandera, I.; Palacios, D.; Barco, R. Traffic Steering for eMBB in Multi-Connectivity Scenarios. Electronics 2020, 9, 2063. [CrossRef]

33. Fikri, A.; Erol, H. Mean squared error matrix comparisons of some biased estimators in linear regression. Commun. -Stat.-Theory Methods 2003, 32, 2389-2413.

34. Muñoz, P.; de-la-Bandera, I.; Ruiz, F.; Luna, S.; Barco, R.; Toril, M.; Lázaro, P.; Rodríguez, J. Computationally-Efficient Design of a Dynamic System-Level LTE Simulator. Int. J. Electron. Telecommun. 2011, 57, 347-358. [CrossRef]

35. ICT-317669-METIS/D6.1, Mobile and Wireless Communications Enablers for the Twenty-Twenty Information Society (METIS). Available online: https:/ / metis2020.com/wp-content/uploads/deliverables/METIS_D6.1_v1.pdf (accessed on 10 May 2021).

36. 3GPP. TR 38.901, Study on Channel Model for Frequencies from 0.5 to $100 \mathrm{GHz}$; Rel-16. Available online: https:/ / portal.3gpp. org/desktopmodules/Specifications/SpecificationDetails.aspx?specificationId=3173 (accessed on 2 May 2021).

37. Rhee, J.; Holtzman, J.M.; Kim, D. Scheduling of real/non-real time services: Adaptive EXP/PF algorithm. In Proceedings of the 57th IEEE Semiannual Vehicular Technology Conference, VTC 2003-Spring, Jeju, Korea, 22-25 April 2003; Volume 1, pp. 462-466. 